\title{
Patient-Reported Outcomes of an Integrative Pain Management Program Implemented in a Primary Care Safety Net Clinic: a Quasi-experimental Study
}

\author{
Maria Teresa Chao, DrPH, MPA ${ }^{1,2}$, Emily Hurstak, MD, MPH, MAS ${ }^{2}$, Kristina Leonoudakis-Watts, $B A^{3}$, \\ Frank Sidders, $\mathrm{MPH}^{3}$, Joseph Pace, $\mathrm{MD}^{3}$, Hali Hammer, $\mathrm{MD}^{3,4}$, and Barbara Wismer, $\mathrm{MD}, \mathrm{MPH}^{3,4}$ \\ 'Osher Center for Integrative Medicine, University of California, San Francisco, San Francisco, CA, USA; ${ }^{2}$ Division of General Internal Medicine at \\ Zuckerberg San Francisco General Hospital and Trauma Center, UCSF, San Francisco, CA, USA; ${ }^{3}$, San Francisco Department of Public Health, San \\ Francisco, CA, USA; ${ }^{4}$ Department of Family and Community Medicine, UCSF, San Francisco, CA, USA.
}

KEY WORDS: chronic pain; integrative medicine; multidisciplinary pain management; multimodal care; primary care; underserved populations; vulnerable populations.

J Gen Intern Med 34(7):1105-7

DOI: $10.1007 / \mathrm{s} 11606-019-04868-0$

(๑) Society of General Internal Medicine 2019

\section{INTRODUCTION}

Socioeconomically vulnerable patients experience high rates of pain, barriers to care, ${ }^{1}$ and risks from conventional pain treatments. ${ }^{2}$ Multimodal, non-pharmacologic approaches are recommended as first-line treatments for chronic pain, ${ }^{3}$ but are often unavailable in resourcelimited settings. We evaluated the impact of a multimodal chronic pain management program among patients at a safety-net clinic.

\section{METHODS}

Setting and Intervention. We developed the Integrative Pain Management Program (IPMP) at Tom Waddell Urban Health Clinic (TWUHC), a public health, primary care clinic in San Francisco. TWUHC provides healthcare for diverse, marginalized patients with high rates of homelessness, chronic disease, chronic pain, and substance use. IPMP consists of a core 12-week program centered around a weekly "home group" that provides education on the biopsychosocial model of pain and multimodal treatments, physical movement, mindfulness training, and peer support. Patients also receive acupuncture, massage, and health coaching. After the core program, IPMP patients have ongoing access to weekly groups, offering peer support, education, mindfulness, movement, and acupuncture.

Design, Participants, and Measures. We evaluated IPMP using a quasi-experimental design comparing IPMP participants vs. eligible, interested patients who had not yet

Published online February 19, 2019 enrolled in IPMP. Participants were referred by a primary care provider. Eligible patients reported pain $>3$ months and were prescribed opioids. We excluded patients who were non-English speaking, pregnant, or unable to participate in groups. IPMP participants completed validated surveys of pain-related outcomes ${ }^{4,5}$ at baseline, 3 months, and 6 months. Eligible patients who were interested in IPMP but could not immediately enroll due to scheduling or other barriers were invited to complete surveys at baseline and 3 months, forming a non-randomized comparison group.

Analysis. We used linear mixed models (LMMs) to analyze changes in patient-reported outcomes from baseline to 3- and 6-month follow-up. We conducted two sets of analyses using LMMs: changes in outcomes for IPMP participants vs. comparison group; and changes in outcomes of IPMP participants who attended $<8$ home groups vs. $\geq 8$ home groups.

\section{RESULTS}

Primary care providers referred 146 patients to IPMP; 73 were eligible and interested; 61 consented to research (IPMP participants $n=41$, comparison group $n=20$ ). Participants were low-income and racially/ethnically diverse; $13 \%$ were unstably housed; $96 \%$ reported chronic pain for $>1$ year. Relative to the comparison group, IPMP participants had less optimal mental health and were prescribed lower opioid doses (Table 1).

IPMP participants had statistically significant improvements in pain interference, pain intensity, social satisfaction, global mental health, and pain self-efficacy at 3 and 6 months (Table 2). No statistically significant changes were observed in other measures. We did not observe significant changes in the comparison group for any patient-reported outcomes, except for physical functioning at 3 months. IPMP participants who attended $\geq 8$ home groups $(n=26)$ reported greater improvements in most 
Table 1 Characteristics of Study Participants at Baseline $(n=61)$

\begin{tabular}{|c|c|c|c|}
\hline & \multirow{2}{*}{$\frac{\text { Comparison group }(n=20)}{N(\%) \text { or mean } \pm \text { SD }}$} & \multirow{2}{*}{$\frac{\text { IPMP participants }(n=41)}{N(\%) \text { or mean } \pm \text { SD }}$} & \multirow[t]{2}{*}{$p$ value } \\
\hline & & & \\
\hline Age (years) & $53.2 \pm 9.7$ & $56.0 \pm 7.9$ & 0.23 \\
\hline Female gender & $8(40)$ & $21(51)$ & 0.59 \\
\hline \multicolumn{3}{|l|}{ Race/ethnicity ${ }^{*}$} & 0.12 \\
\hline African American/Black & $7(37)$ & $8(21)$ & \\
\hline Asian or Pacific Islander & - & $1(3)$ & \\
\hline Hispanic/Latino & $3(16)$ & $6(16)$ & \\
\hline White or Caucasian & $3(16)$ & $14(37)$ & \\
\hline Other or more than one race ${ }^{\dagger}$ & $6(31)$ & $9(23)$ & \\
\hline Education, high school or less & $10(50)$ & $19(46)$ & 0.07 \\
\hline Disabled* & $13(68)$ & $31(76)$ & 0.56 \\
\hline Annual household income $<\$ 35 \mathrm{k}^{*}$ & $17(89)$ & $41(100)$ & 0.11 \\
\hline Married or in a relationship & $4(21)$ & $10(24)$ & 0.62 \\
\hline \multicolumn{3}{|l|}{ Housing status } & 0.76 \\
\hline Unstable or transitional housing & $3(15)$ & $5(12)$ & \\
\hline Renting a room & $5(25)$ & $14(34)$ & \\
\hline Renting an apartment or house & $12(60)$ & $22(54)$ & \\
\hline \multicolumn{4}{|l|}{ Risk of problematic substance use ${ }^{*}, \S$} \\
\hline Alcohol & $4(21)$ & $5(14)$ & 0.50 \\
\hline Cannabis & $5(26)$ & $17(47)$ & 0.13 \\
\hline Cocaine & $6(32)$ & $16(44)$ & 0.55 \\
\hline Opioids & $6(32)$ & 7 (19) & 0.31 \\
\hline Post-traumatic stress disorder & $1(5)$ & $15(37)$ & 0.01 \\
\hline Prescription opioid dose (MME)\| & $312.9 \pm 523.4$ & $140.4 \pm 181.6$ & 0.06 \\
\hline \multicolumn{4}{|l|}{ Patient-reported outcomes } \\
\hline Pain interference $e^{\mathbb{T}}$ & $67.12 \pm 5.82$ & $67.38 \pm 4.87$ & 0.86 \\
\hline Average pain intensity ${ }^{\mathrm{g}}$ & $6.95 \pm 2.04$ & $6.72 \pm 1.77$ & 0.66 \\
\hline Anxiety ${ }^{\mathrm{P}}$ & $60.76 \pm 7.59$ & $62.44 \pm 7.08$ & 0.41 \\
\hline Depressive symptoms $^{\mathrm{I}}$ & $56.05 \pm 9.66$ & $60.06 \pm 7.23$ & 0.08 \\
\hline Physical functioning & $35.60 \pm 5.63$ & $35.62 \pm 5.79$ & 0.99 \\
\hline Social satisfaction & $39.93 \pm 9.18$ & $37.70 \pm 7.68$ & 0.34 \\
\hline Global mental health & $40.28 \pm 10.76$ & $34.97 \pm 7.08$ & 0.03 \\
\hline Global physical health & $36.32 \pm 4.35$ & $34.55 \pm 5.35$ & 0.22 \\
\hline Pain catastrophizing ${ }^{\mathrm{dI}}$ & $27.89 \pm 11.80$ & $29.86 \pm 11.68$ & 0.56 \\
\hline Pain self-efficacy & $29.58 \pm 12.76$ & $23.97 \pm 11.69$ & 0.11 \\
\hline
\end{tabular}

${ }^{*}$ Missing data: racelethnicity $=4$, disabled $=1$, annual household income $=1$, relationship status $=1$, risk of problematic substance use $=5$

${ }^{\dagger}$ Participants who reported more than one racelethnicity included 3 African American/Native American, 1 African American/White, 2 Asian/Native American, 2 Asian/White, 4 Native American/White

${ }^{\ddagger}$ Unstable or transitional housing included 1 on the streets, 1 in a homeless shelter, 2 in transitional housing, 3 in single room occupancy hotel, 1 sailboat

${ }^{\S}$ Risk of problematic substance use defined by the National Institute of Drug Abuse's Alcohol Use Disorders Identification Test score $\geq 4$ or Alcohol Smoking Substance Involvement Screening Test (ASSIST) score $\geq 7$

"Average daily opioid dose presented as morphine milligram equivalent (MME)

"Lower score indicates a more optimal outcome

outcomes (Table 2), though differences were not significant when compared to changes in those who attended $<8$ home groups $(n=20)$.

\section{DISCUSSION}

Among a sample of diverse at-risk participants, we observed statistically significant and clinically relevant improvements in pain interference, social satisfaction, and global mental health that were sustained at 6 months. Changes in pain intensity were statistically significant and sustained at 6 months, but not at a magnitude considered clinically relevant. A high proportion of patients in the program have unstable housing and histories of trau- ma, thought to create barriers to engagement with multimodal pain management. Our findings suggest that implementation of a multimodal program may improve patients' quality of life, even for patients known to be medically, psychosocially, and behaviorally complex.

We used a non-randomized design to ensure that patients could enroll in IPMP as soon as possible. A lack of randomization, self-selection, and baseline differences between IPMP participants and the comparison group may have biased our findings. Compared with non-participants, IPMP participants had lower overall opioid doses and may have been motivated to participate in IPMP due to unresolved pain, recent opioid tapering, or interest in nonpharmacologic options. Generalizability may be limited because IPMP was supported by institutional funding 
Table 2 Patient-Reported Outcomes, Change From Baseline by Group*

\begin{tabular}{|c|c|c|c|c|}
\hline & \multirow{2}{*}{$\begin{array}{l}\text { Comparison group }{ }^{\dagger}(n= \\
\text { 20) }\end{array}$} & \multirow{2}{*}{$\begin{array}{l}\text { IPMP participants }{ }^{\dagger}(n= \\
\text { 41) }\end{array}$} & \multicolumn{2}{|c|}{ IPMP Participants only } \\
\hline & & & $<8$ sessions $(n=20)$ & $\geq 8$ sessions $(n=26)$ \\
\hline Pain interference $\Delta$ at 3 months $^{\S}$ & $-0.98(-4.43,2.46)$ & $-2.59(-4.53,-0.64)$ & $-0.87(-3.80,2.06)$ & $\begin{array}{l}-3.85(-6.18 \\
-1.53)\end{array}$ \\
\hline$\Delta$ at 6 months $^{\S}$ & & $-3.81(-5.90,-1.71)$ & $\begin{array}{l}-3.73(-7.21 \\
-0.26)\end{array}$ & $\begin{array}{l}-4.48(-6.96 \\
-2.01)\end{array}$ \\
\hline $\begin{array}{l}\text { Average pain intensity } \Delta \text { at } \\
3 \text { months } \mathrm{s}^{8}\end{array}$ & $-0.01(-0.91,0.89)$ & $-0.78(-1.27,-0.29)$ & $-0.77(-1.58,0.04)$ & $-0.54(-1.18,0.10)$ \\
\hline$\Delta$ at 6 months $^{\S}$ & & $-1.00(-1.53,-0.47)$ & $\begin{array}{l}-1.41(-2.38 \\
-0.45)\end{array}$ & $\begin{array}{l}-0.71(-1.40 \\
-0.03)\end{array}$ \\
\hline Anxiety $\Delta$ at 3 months $^{\S}$ & $2.76(-1.82,7.34)$ & $-2.25(-4.81,0.31)$ & $-1.34(-5.48,2.80)$ & $\begin{array}{l}-3.88(-7.17 \\
-0.58)\end{array}$ \\
\hline$\Delta$ at 6 months ${ }^{\S}$ & & $-1.07(-3.83,1.69)$ & $-0.46(-5.39,4.47)$ & $-2.12(-5.64,1.39)$ \\
\hline $\begin{array}{l}\text { Depressive symptoms } \Delta \text { at } \\
3 \text { months }^{\S}\end{array}$ & $2.19(-2.29,6.68)$ & $-1.97(-4.47,0.52)$ & $1.92(-1.69,5.52)$ & $\begin{array}{l}-4.79(-7.65 \\
-1.94)\end{array}$ \\
\hline$\Delta$ at 6 months $^{\S}$ & & $-1.29(-3.97,1.40)$ & $-2.37(-6.66,1.92)$ & $-1.80(-4.85,1.25)$ \\
\hline $\begin{array}{l}\text { Physical functioning } \Delta \text { at } 3 \text { months } \\
\Delta \text { at } 6 \text { months }\end{array}$ & $3.64(0.84,6.43)$ & $\begin{array}{l}0.17(-1.37,1.71) \\
0.04(-1.62,1.70)\end{array}$ & $\begin{array}{l}0.58(-1.79,2.95) \\
1.91(-0.91,4.73)\end{array}$ & $\begin{array}{l}-0.64(-2.50,1.23) \\
-1.12(-3.12,0.88)\end{array}$ \\
\hline $\begin{array}{l}\text { Social satisfaction } \Delta \text { at } 3 \text { months } \\
\Delta \text { at } 6 \text { months }\end{array}$ & $0.58(-3.94,5.10)$ & $3.06(0.51,5.61)$ & $2.49(-1.31,6.30)$ & $2.68(-0.28,5.63)$ \\
\hline 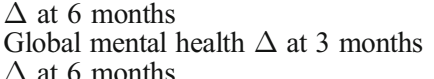 & $0.32(-3.64,4.29)$ & $\begin{array}{l}3.56(0.82,6.31) \\
2.76(0.57,4.94) \\
3.64(128,600)\end{array}$ & $\begin{array}{l}6.06(1.53,10.60) \\
0.45(-2.20,3.10) \\
108(-2.074 .24)\end{array}$ & $\begin{array}{l}2.30(-0.86,5.45) \\
2.46(0.35,4.57) \\
1.37(-0.88 .361)\end{array}$ \\
\hline $\begin{array}{l}\text { Global physical health } \Delta \text { at } \\
\text { months }\end{array}$ & $-0.59(-3.67,2.49)$ & $\begin{array}{l}3.64(1.28,6.00) \\
1.98(0.26,3.70)\end{array}$ & $\begin{array}{l}1.08(-2.07,4.24) \\
1.17(-1.94,4.28)\end{array}$ & $\begin{array}{l}1.37(-0.88,3.61) \\
3.38(0.92,5.84)\end{array}$ \\
\hline $\begin{array}{l}\Delta \text { at } 6 \text { months } \\
\text { Pain catastrophizing } \Delta \text { at } 3 \text { months }{ }^{\S} \\
\Delta \text { at } 6 \text { months }^{\S}\end{array}$ & $-0.08(-5.71,5.54)$ & $\begin{array}{l}1.37(-0.48,3.22) \\
-1.29(-4.36,1.79) \\
-4.78(-8.10,-1.46)\end{array}$ & $\begin{array}{l}5.37(1.67,9.07) \\
-1.8(-6.57,2.96) \\
-3.46(-9.13,2.20)\end{array}$ & $\begin{array}{l}2.99(0.36,5.62) \\
-1.92(-5.66,1.81) \\
-5.83(-9.83, \\
-1.83)\end{array}$ \\
\hline $\begin{array}{l}\text { Pain self-efficacy } \Delta \text { at } 3 \text { months } \\
\Delta \text { at } 6 \text { months }\end{array}$ & $-2.11(-9.07,4.85)$ & $\begin{array}{l}5.15(1.31,9.00) \\
7.37(3.22,11.52)\end{array}$ & $\begin{array}{l}5.67(-0.27,11.62) \\
10.63(3.55,17.71)\end{array}$ & $\begin{array}{l}5.29(0.58,10.00) \\
6.39(1.36,11.42)\end{array}$ \\
\hline
\end{tabular}

${ }^{*}$ Data derived from linear mixed models. $p<0.05$ statistically significant changes from baseline are in italic

"“Comparison group" refers to eligible and interested patients who had not yet attended IPMP; follow-up data collected only at 3 months because some participants then began the IPMP program. Data include baseline $(n=20)$ and 3-month follow-up $(n=12)$. "IPMP participants" refers to patients who attended at least one IPMP group session, excluding 5 waitlist participants who later attended IPMP

"Analysis of "IPMP Participants Only" compares those who attended $<8$ group sessions vs. $\geq 8$ group sessions, including 5 participants who were initially on a waitlist. Data include baseline $(n=46)$, 3-month follow-up $(n=37)$, and 6-month follow-up $(n=28)$

${ }^{\xi}$ Lower score indicates a more optimal outcome

and in-kind staff support not available in all settings. Nonetheless, our study contributes to the sparse literature on integrative pain approaches in safety net settings. ${ }^{6} \mathrm{We}$ provide practice-based data suggesting that increasing access to non-pharmacologic approaches in primary care safety-net settings may be a strategy to improve pain management for vulnerable patients with chronic pain.

Acknowledgments: The authors thank Blue Walcer, MPH, for her involvement with program development, facilitation of weekly groups, and service on the IPMP Steering Committee; Jimmy He for assistance coordinating and conducting interviews, monitoring and collecting attendance, and quality improvement data; and Trilce Santana for conducting interviews of participants and providing training and support to IPMP staff for best practices in research conduct. Institutional support for IPMP was provided through the San Francisco Department of Public Health.

Corresponding Author: Maria Teresa Chao, DrPH, MPA; Osher Center for Integrative Medicine, University of California, San Francisco, San Francisco, CA, USA (e-mail: Maria.Chao@ucsf.edu).

Funding Information Funding for the study was provided through the Mount Zion Health Fund.

\section{Compliance with Ethical Standards:}

Conflict of Interest: The authors declare that they do not have a conflict of interest.

Publisher's Note: Springer Nature remains neutral with regard to jurisdictional claims in published maps and institutional affiliations.

\section{REFERENCES}

1. Institute of Medicine. Relieving Pain in America: A Blueprint for Transforming Prevention, Care, Education, and Research. Washington, DC: The National Academies Press; 2011.

2. Song Z. Mortality Quadrupled Among Opioid-Driven Hospitalizations, Notably Within Lower-Income And Disabled White Populations. Health Affairs. 2017;36(12):2054-61. https://doi.org/10.1377/hlthaff.2017.0689

3. Dowell D, Haegerich TM, Chou R. CDC Guideline for Prescribing Opioids for Chronic Pain-United States, 2016. JAMA. 2016;315(15):1624-45. https://doi.org/10.1001/jama.2016.1464

4. Amtmann D, Cook KF, Jensen MP, et al. Development of a PROMIS item bank to measure pain interference. Pain. 2010;150(1):173-82. doi:https:// doi.org/10.1016/j.pain.2010.04.025

5. Nicholas MK. The pain self-efficacy questionnaire: Taking pain into account. Eur J Pain. 2007;11(2):153-63. https://doi.org/10.1016/j. ejpain.2005.12.008

6. Hurstak E, Kushel MB. Pain Care on a New Track: Complementary Therapies in the Safety Net. California Health Care Foundation; 2016. 